\title{
A THEOREM ON INJECTIVITY OF THE CUP PRODUCT
}

\author{
JOHN C. WOOD ${ }^{1}$
}

\begin{abstract}
ABSTRACr. We prove that if a space $X$ has abelian or sufficiently abelian fundamental group, then the cup product $H^{1}(X) \wedge H^{1}(X) \rightarrow$ $H^{2}(X)$ is injective, giving an inequality between the associated Betti numbers. This generalises to a theorem of injectivity of the $k$-fold cup product on $H^{n}(X)$, given that the $k$ th order Whitehead product on $\pi_{n}(X)$ is trivial or torsion.
\end{abstract}

Let $X$ be a topological space, and let $H_{n}(X)$ denote its $n$th singular integral homology group. Let $\mathscr{A}$ denote the class of groups $\pi$ such that $p: \pi \rightarrow \pi /[\pi, \pi]$ splits rationally, i.e. there exists a homomorphism $q: \pi /[\pi, \pi] \rightarrow \pi$ such that $p q \otimes 1: \pi /[\pi, \pi] \otimes Q \rightarrow \pi /[\pi, \pi] \otimes Q$ is an isomorphism.

Set $\odot^{k} H^{n}(X)$ equal to, if $n$ is odd, the $k$ th exterior power $\wedge^{k} H^{n}(X)$, and if $n$ is even, the $k$ th symmetric power of $H^{n}(X)$, then:

THEOREM 1. The cup product pairing: $\odot{ }^{k} H^{n}(X) \rightarrow H^{k n}(X)$ is injective $(n \geqq 1, k \geqq 2)$ if:

(a) the Hurewicz homomorphism: $\pi_{n}(X) \rightarrow H_{n}(X)$ is epimorphic,

(b) $H_{n-1}(X)$ is a free $Z$-module,

(c) $($ for $(n, k) \neq(1,2))$ the kth order Whitehead product: $\pi_{n}(X) \times \cdots \times$ $\pi_{n}(X) \rightarrow \pi_{k n-1}(X)$ is trivial or torsion (i.e. each Whitehead product contains zero or a torsion element),

$\left(c^{\prime}\right)($ for $(n, k)=(1,2)) \pi_{1}(X) \in \mathscr{A}$.

Note. The class $\mathscr{A}$ includes abelian groups, periodic groups, and also such nearly abelian groups as the group generated by $a, b$ under the single relation: $a^{n} b=b a^{n}$, this being $\pi_{1}$ of the space $\left(S_{1}^{1} \vee S_{2}^{1}\right) \cup_{\left[n i_{1}, i_{2}\right]} e^{2}$.

COROLlaRY 1. Under the hypotheses of Theorem 1, the Betti numbers $\beta_{r}$ satisfy the following inequality:

$$
\left(\begin{array}{c}
\beta_{n}+k-1 \\
k
\end{array}\right) \leqq \beta_{k n}, \quad n \text { even, } \quad\left(\begin{array}{c}
\beta_{n} \\
k
\end{array}\right) \leqq \beta_{k n}, \quad n \text { odd }
$$

Received by the editors January 10, 1972 and, in revised form, April 25, 1972.

AMS (MOS) subject classifications (1970). Primary 55B45; Secondary 55E15, 57A65.

Key words and phrases. Fundamental group, Whitehead products, cup products, Betti numbers.

${ }^{1}$ During the period of this research, the author was supported by Science Research Council grant B-70-809.

(c) American Mathematical Society 1973 
Corollary 2. If $\pi_{1}(X) \in \mathscr{A}$, the cup product: $H^{1}(X) \wedge H^{1}(X) \rightarrow H^{2}(X)$ is injective, and thus $\frac{1}{2} \beta_{1}\left(\beta_{1}-1\right) \leqq \beta_{2}$.

Corollary 2 was proved by K.-T. Chen [1] for a differentiable manifold with abelian fundamental group, using real or complex deRham cohomology. A proof valid for CW complexes with finitely generated homotopy groups and cohomology coefficients in any field is given by Massey in a footnote to [1]. The inequality on the Betti numbers was first noted by Hopf [2] for a simplicial complex with abelian fundamental group.

Corollary 3. If $X$ is a compact connected 3-manifold with $\pi_{1}(X) \in \mathscr{A}$, then $\beta_{1} \leqq 3$.

K. Reidemeister proved this for $\pi_{1}(X)$ abelian in [3].

COROllaRY 4. If $X$ is a compact connected orientable 4-manifold with $\pi_{1}(X) \in \mathscr{A}$, then the Euler number of $X \geqq-1$.

Proof of Theorem 1. We use the following Lemma whose proof is omitted:

Lemma. Let $E$ be a $Z$-module, and let $x^{1}, \cdots, x^{m}$ be linearly independent elements of $\operatorname{Hom}(E, Z)$. Then there exists a positive integer $K$, linearly independent elements $y^{1}, \cdots, y^{m} \in \operatorname{Hom}(E, Z)$, and $e_{1}, \cdots, e_{m} \in E$, such that $K x^{1}, \cdots, K x^{\prime n}$ are linearly equivalent ${ }^{2}$ to $y^{1}, \cdots, y^{m}$, and $\left\langle y^{i}, e_{j}\right\rangle=$ $K \delta_{i j}$.

We proceed to the proof of Theorem 1 .

By hypothesis (b), $H^{n}(X) \cong \operatorname{Hom}\left(H_{n}(X), Z\right)$. Suppose $x^{1}, \cdots, x^{m}$ are linearly independent elements of $H^{n}(X)$. By the Lemma there exists integer $K, y^{1}, \cdots, y^{m} \in H^{n}(X)$, linearly equivalent to $K x^{1}, \cdots, K x^{m}$, and $e_{1}, \cdots, e_{m} \in H_{n}(X)$ such that $\left\langle y^{i}, e_{j}\right\rangle=K \delta_{i j}$; to prove injectivity of the cup product, it suffices to prove that the elements

$$
y^{i_{1}} \cup \cdots \cup y^{i_{k}}, \quad 1 \leqq i_{1}<\cdots<i_{k} \leqq m
$$

(with < replaced by $\leqq$ when $n$ is even) are linearly independent in $H^{k n}(X)$.

We choose an integer $P$ and elements $a_{1}, \cdots, a_{m} \in \pi_{n}(X)$ with homology classes $P e_{1}, \cdots, P e_{m}$ such that each Whitehead product $\left[a_{i_{1}}, \cdots, a_{i_{k}}\right]$ contains zero, as follows:

If $(n, k)=(1,2)$ choose $a_{1}^{\prime}, \cdots, a_{m}^{\prime} \in \pi /[\pi, \pi] \otimes Q$ such that $p q \otimes 1\left(a_{i}^{\prime}\right)$ has homology class $e_{i} \otimes 1$, choose $P$ so that each $P a_{i}^{\prime}$ actually lies in $\pi /[\pi, \pi]$ and set $a_{i}=q\left(P a_{i}^{\prime}\right)$.

\footnotetext{
${ }^{2}$ I.e. each set is linearly dependent on the other.
} 
If $(n, k) \neq(1,2)$ choose $a_{1}^{\prime}, \cdots, a_{m}^{\prime} \in \pi_{n}(X)$ with homology classes $e_{1}, \cdots, e_{m}$. By hypothesis, each Whitehead product $\left[a_{i_{1}}^{\prime}, \cdots, a_{i_{k}}^{\prime}\right]$ contains a torsion element. Let $P$ be chosen so that $0 \in P\left[a_{i_{1}}^{\prime}, \cdots, a_{i_{k}}^{\prime}\right]$ for all $i_{1}, \cdots, i_{k}$. Set $a_{i}=P a_{i}^{\prime}$. It follows from [4] that $0 \in\left[a_{i_{1}}, \cdots, a_{i_{k}}\right]$.

Form the wedge of maps representing $a_{i_{1}}, \cdots, a_{i_{k}}: S^{n} \vee \cdots \vee S^{n} \rightarrow X$; then according to Porter [4, Theorem 2.4], hypothesis (c) allows us to extend to a map $k: S^{n} \times \cdots \times S^{n} \rightarrow X$.

By naturality $k^{*}\left(y^{j_{1}} \cup \cdots \cup y^{j_{k}}\right)=k^{*} y^{j_{1}} \cup \cdots \cup k^{*} y^{j_{k}}$. Evaluating the $k^{*} y^{j_{i}}$ on the generators of $H_{n}\left(S^{n} \times \cdots \times S^{n}\right)$ we have:

$$
\begin{aligned}
k^{*}\left(y^{j_{1}} \cup \cdots \cup y^{j_{k}}\right) & =0 & & \left(j_{1}, \cdots, j_{k}\right) \neq\left(i_{1}, \cdots, i_{k}\right), \\
& =(K P)^{k} M & & \left(j_{1}, \cdots, j_{k}\right)=\left(i_{1}, \cdots, i_{k}\right),
\end{aligned}
$$

with $M=1$ if is odd; or $M=\left|N_{1}\right| ! \cdots\left|N_{t}\right|$ !, if $n$ is even, where $N_{1}, \cdots$, $N_{t}$ is a partition of the indices $1, \cdots, k$ under the equivalence relation $r \sim s$ if and only if $j_{\tau}=j_{s}$. This shows that the coefficient of each $x^{i_{1}} \cup \cdots \cup$ $x^{i_{k}}$ in any relation of linear dependence of the elements (i) must be zero, hence injectivity of the cup product is established.

Notes. (1) If $R$ is a ring which is torsion free as a $Z$-module, then under the hypotheses of Theorem 1 , the universal coefficient formula shows that the cup product: $\bigodot^{k} H^{n}(X ; R) \rightarrow H^{k n}(X ; R)$ is injective. When $R=Q$, a slight modification of the proof of Theorem 1 using $H^{n}(X, Q)=$ $\operatorname{Hom}\left(H_{n}(X, Z), Q\right)$ shows that hypothesis (b) is unnecessary for injectivity: $\bigodot^{k} H^{n}(X, \boldsymbol{Q}) \rightarrow H^{k n}(X, \boldsymbol{Q})$. Note this shows Corollary 1 is true without hypothesis (b).

(2) The theorem is, in spirit, the contrapositive of Theorem 3.3 of [4], and uses a similar calculation in its proof. The essential idea is that a Whitehead product which is trivial or torsion forces a cup product to be nonzero. As an example of how we can create a cup product by killing a Whitehead product, let $X$ be the space formed by attaching a $2 n$-cell to $S^{n}$ ( $n$ even) via the Whitehead square of the generator of $\pi_{n} S^{n}$. If $x$ is the generator of $H^{n}(X)$, a simple calculation similar to that used in Theorem 1 shows $x \cup x=$ twice the generator of $H^{2 n}(X)$. The opposite process of creating a Whitehead product by killing a cup product is expounded by Porter in [5, Theorem 3.15].

(3) To obtain a version of Theorem 1 for coefficients in $Z_{p}, p$ prime, we must replace hypothesis (a) by the assumption that the composite: $\pi_{n}(X) \rightarrow H_{n}(X) \rightarrow H_{n}\left(X ; Z_{p}\right)$ is surjective, remove hypothesis (b), and replace hypothesis (c) by:

(if $(n, k)=(1,2)) p: \pi \rightarrow \pi /[\pi, \pi]$ splits $\bmod p$, i.e. there exists $q: \pi /[\pi, \pi] \rightarrow$ $\pi$ such that $p q \otimes 1: \pi /[\pi, \pi] \otimes Z_{p} \rightarrow \pi /[\pi, \pi] \otimes Z_{p}$ is an isomorphism,

(if $(n, k) \neq(1,2))$ each Whitehead product $\left[a_{1}, \cdots, a_{k}\right]$, where $a_{i} \in \pi_{n}(X)$, contains a torsion element of order prime to $p$. 
We conclude that the cup product: $\odot{ }^{k} H^{n}\left(X ; Z_{p}\right) \rightarrow H^{k n}\left(X ; Z_{p}\right)$ is injective provided either $n$ is odd or $n$ is even and $k<p$. This last restriction ensures that the $M$ in the proof of Theorem 1 is nonzero $\bmod p$, and its necessity is shown by the example in note (2) for $k=2, p=2$.

(4) A similar theorem asserting that the cup product is injective on a suitable factor space of $H^{n_{1}}(X) \otimes \cdots \otimes H^{n_{k}}(X)$ can be proved under the hypotheses (a), (b) of Theorem 1 for each $n=n_{i}$, and the hypothesis that the $k$ th order Whitehead product:

$$
\pi_{n_{1}}(X) \times \cdots \times \pi_{n_{k}}(X) \rightarrow \pi_{n_{1}+\cdots+n_{k}-1}(X)
$$

is trivial or torsion.

In conclusion I should like to thank Professor Jim Eells for drawing my attention to this problem, Dr. Alan Thomas for some useful conversations on the subject, and the referee for his valuable suggestions for improvement of the original manuscript.

\section{REFERENCES}

1. K.-T. Chen, A sufficient condition for nonabelianness of fundamental groups of differentiable manifolds, Proc. Amer. Math. Soc. 26 (1970), 196-198. MR 43 \#5543.

2. H. Hopf, Fundamentalgruppe und zweite Bettische Gruppe, Comment. Math. Helv. 14 (1942), 257-309. MR 3, 316.

3. K. Reidemeister, Kommutative Fundamentalgruppen, Monatsch. Math. Phys. 43 (1936), 20-28.

4. G. J. Porter, Higher-order Whitehead products, Topology 3 (1965), 123-135. MR 30 \#4261.

5. —_, Spaces with vanishing Whitehead products, Quart. J. Math. Oxford Ser. (2) 16 (1965), 77-84. MR 30 \#2511.

Department of Mathematics, University of Warwick, Coventry, England 